\title{
Metabolomics in Chronic Kidney Diseases: Here to Stay
}

\author{
Mihaela-Roxana Pătruică ${ }^{1,2,3}$, Florica Gădălean ${ }^{1,2}$, Adrian Vlad ${ }^{2,4}$, Adrian Sturza ${ }^{3,5}$, \\ Dragos Cătălin Jianu ${ }^{2,6,7}$, Lavinia Balint ${ }^{1,2}$, Silvia Ienciu ${ }^{1}$, Ligia Petrica ${ }^{1,2,3, *}$, \\ Danina M. Muntean ${ }^{2,3,5, *}$ and Carmen Socaciu ${ }^{2,8}$
}

\footnotetext{
${ }^{\text {I }}$ Department of Internal Medicine II-Nephrology, Victor Babeș University of Medicine and Pharmacy Timișoara, 2 Eftimie Murgu Sq., 30004I Timișoara, Romania; mihaelapatruica@gmail.com (M.-R.P.); fnnicorici@yahoo.com (F.G.); balintlavi@yahoo.com (L.B.); ienciu_silviaoana@yahoo.com (S.I.)

${ }^{2}$ Centre for Molecular Research in Nephrology and Vascular Disease, Victor Babeș University of Medicine and Pharmacy Timișoara, 2 Eftimie Murgu Sq., 30004I Timișoara, Romania; vlad.adrian@umft.ro(A.V.); jianu.dragos@umft.ro(D.C.J.); carmen.socaciu@usamvcluj.ro (C.S.)

${ }_{3}$ Center for Translational Research and Systems Medicine, Victor Babes University of Medicine and Pharmacy

Timişoara, 2 Eftimie Murgu Sq., 30004I Timișoara, Romania; sturza.adrian@umft.ro

${ }^{4}$ Department of Internal Medicine II-Diabetes and Metabolic Diseases, Victor Babeș University of Medicine and

Pharmacy Timișoara, 2 Eftimie Murgu Sq., 30004I Timișoara, Romania

${ }^{5}$ Department of Functional Sciences-Pathophysiology, Victor Babeș University of Medicine and Pharmacy

Timişoara, 2 Eftimie Murgu Sq., 30004I Timişoara, Romania

${ }^{6}$ Department of Neurosciences-Neurology, Victor Babeș University of Medicine and Pharmacy Timișoara,

2 Eftimie Murgu Sq., 30004I Timișoara, Romania

7 Centre for Cognitive Research in Neuropsychiatric Pathology, Victor Babeș University of Medicine and Pharmacy

Timişoara, 2 Eftimie Murgu Sq., 30004I Timișoara, Romania

${ }^{8}$ Department of Food Science, Faculty of Food Science and Technology, University of Agricultural Sciences and

Veterinary Medicine Cluj-Napoca, 400372 Cluj-Napoca, Romania

*Correspondence: petrica.ligia@umft.ro (L.P.); daninamuntean@umft.ro (D.M.M.)
}

Submitted: Io December 2020; Accepted: 4 February 202I; Published: I6 February 202I

\begin{abstract}
Chronic kidney disease (CKD) affects around $13 \%$ of the adult population, has become a considerable concern worldwide, and is characterized by inadequate renal clearance, resulting in the accumulation of various potential toxic compounds. Metabolomics, one of the many important parts of "omics" science, refers to the systematic study of metabolites in a living system and their changes because of pathophysiological and genetic modifications. The use of metabolomics in the nephrology field of research has offered a better understanding of the pathomechanisms of CKD. The most recent technologies used for the evaluation of plasma and urinary metabolites are nuclear magnetic resonance (NMR) and mass spectroscopy (MS). A major research direction of modern medicine is to develop new therapies and new biomarkers for the early diagnosis of patients with CKD. Experimental studies of renal metabolism unequivocally demonstrated that kidney function has a huge impact on several circulating metabolites. Moreover, metabolites themselves appear to have an essential role in CKD pathogenesis/complications, being recognized as potential biomarkers with prognostic, diagnostic, and therapeutic value for the disease. The present paper provides a bird's eye view on metabolomics and a summary of the most recent studies of metabolomics carried out in patients with CKD.
\end{abstract}

Keywords: metabolomics; biomarkers; chronic kidney disease 
How to cite: Pătruică, M.-R.; Gădălean, F.; Vlad, A.; Sturza, A.; Jianu, D.C.; Balint, L.; Ienciu, S.; Petrica, L.; Muntean, D.M.; Socaciu, C. Metabolomics in Chronic Kidney Diseases: Here to Stay. Timisoara Med. 2020, 2020(2), 8; doi:10.35995/tmj20200208.

\section{Introduction}

Over the past decade, a series of new technologies have been developed to assess and characterize thousands of macromolecules and small molecules within tissue or body fluids. The scientific field affiliated with the identification of these biological molecules is called "omics".

The omics platforms include genomics (the analysis of genes), transcriptomics (mRNA), proteomics (proteins), lipidomics (lipids), epigenomics (methylated DNA, histone modification and RNA associated silencing), and metabolomics (metabolites). In the era of personalized medicine, a common aim is to identify and characterize biological patterns that help to understand various pathophysiological processes. The main goals of omics-based measurements are to develop new techniques allowing for an early diagnosis and/or a prognostic value of a specific disease, concomitantly with identifying novel metabolic/signal transduction pathways as therapeutic targets [I].

This summary addresses the role of metabolomics in the pathophysiology of chronic kidney disease (CKD). At the same time provides an update on the most recent clinical and experimental renal metabolomics studies.

\section{Metabolomics}

The global increase in life expectancy rate at birth is associated with the rise of chronic diseases and conditions, displaying a huge impact on economics through the aging of the population. To date, the estimation of chronic diseases by the Global Burden of Disease (GBD) accounts for $54 \%$, predicting that by 2030 s the number of all chronic conditions will rise to $65 \%$ [2].

Currently, the healthcare systems worldwide are facing an unprecedented growth of metabolic, cardiac, and renal pathologies, including obesity, diabetes, osteoporosis, hypertension, atherosclerosis, and CKD. Of note, the major challenges of developed countries are those related to the deranged metabolism rather than to nutritional deficiencies. Each chronic disease has a classic biomarker as a hallmark of a disrupted biochemical pathway, such as cholesterol for cardiovascular disease, glucose for diabetes mellitus, and serum creatinine for CKD. All these biochemical pathways are a part of an integrative system [3].

The elucidation of the human genome allowed the development of novel research strategies aimed at understanding the relationship between phenotype and genomics [4]. Metabolomics is the reflection of phenotype and highly responsive to environmental and biological regulatory mechanisms, with a special approach to characterize the phenotype. Modern analytical platforms, such as mass spectrometry, chromatographic systems, and NMR (nuclear magnetic resonance spectroscopy), can produce simultaneously high content data and quantitative data on many metabolites. The next big step of modern medicine is to develop additional technologies capable to evaluate low abundance metabolites, including bioactive products that act in signaling functions and have a low tissue concentration [5]. 
Metabolomics or metabolite profiling, one of the many important parts of "omics" science, is a systematic study used to quantify and identify exogenous and endogenous small molecules $(<\mathrm{rDa})$ or metabolites in a biological system (cells, biofluids, or tissues). The data obtained have pre-clinical applications in toxicology, molecular biology, and personalized medicine based on clinical chemistry [6].

\section{Metabolites' Profiling}

Cells produce molecules known as metabolites. Biological samples such as lipids, amino acids, nucleosides, exogenous substances (drugs and their metabolites), metabolic intermediates in carbohydrate biochemical pathways, hormones, and other signaling molecules are the source of metabolites. Metabolite profiles are estimated at 3000-20,000 under a specific environmental, nutritional, and genetic condition. Interestingly, among other parts of "omics", metabolomics is an important source for discovering new biomarkers [7].

\section{A Historical Perspective}

Roger Williams demonstrated his theory that each individual has a particular "metabolic profile" reflected in the biological fluids in the late 1940s. Several decades later, Horning and his team showed that the gas chromatography-mass spectrometry (GC-MS) technique measures biofluid components. In 1971, the first metabolite was discovered; in the same year, the "metabolomic profile" was observed, while the term "metabolome" was firstly used in 1998 . The first definition of "metabolomics" was elaborated in 1999, and it stated that metabolomics refers to the "quantitative measurement of a dynamic multiparametric metabolic response of living systems to genetic modification or pathophysiological stimuli” [8].

A wide variety of chemical compounds (e.g., organic acids, amino acids, lipids, steroids, carbohydrates, and nucleotides) remained unknown or are not validated, thus the exact number of metabolites is unknown. The total number of the metabolites given by the Human Metabolome Database is II4,098 small molecules that include abundant $(>\mathrm{I} \mu \mathrm{M})$ or rare $(<\mathrm{I} \mathrm{nM})$ metabolites and water-soluble and lipid-soluble metabolites, representing only $20 \%$ of the total metabolome number [9].

\section{Targeted vs. Non-Targeted Analyses}

Analytical strategies for metabolomics are divided into "untargeted-discovery-global" (non-targeted methods) and "targeted-validation-tandem" (targeted methods). A non-targeted approach aims to simultaneously measure all known and unknown metabolites from a biological sample (urine, plasma, or tissue), concomitantly maintaining its ability to quantify the metabolites. Advanced techniques are necessary for a non-targeted approach. Liquid chromatography followed by mass spectrometry (LC/MS) is the standard method used in non-targeted metabolite profiling. By using techniques like LC/MS, thousands of metabolites are identified from biological samples. Nevertheless, mass spectrometry (MS) and nuclear magnetic resonance spectroscopy (NMR) are techniques used in non-targeted metabolomics [Io].

In addition, non-targeted metabolomics has offered us a better comprehension of cellular metabolism, resolved a series of biomedical issues, and has provided novel drug targets by identifying altered metabolic pathways. The disadvantage of non-targeted metabolomics is the possibility of false identification of a metabolite. This innovative concept is called "therapeutic metabolomics" [Io].

In contrast, the targeted metabolomics is a semi-quantitative or a quantitative evaluation a specific set of metabolites (e.g., lipids, amino acids, fatty acids, and sugars) used to comprehend the multitude of metabolic pathways [II]. Commonly, targeted metabolomics has a specific hypothesis. There are many analytical 
tools available for targeted metabolomics (ultraviolet-visible spectroscopy, flame isolation, MS, and NMR). These latter two techniques have high specificity and quantitative reproducibility [ıo].

\section{Metabolomics Technologies}

The large variability of the endogenous metabolites, with a significant difference in size and polarity, led to the development of various methods of identification. The workhorses used in metabolite profiling are nuclear magnetic resonance (NMR) spectroscopy and mass spectrometry (MS), the last using gas chromatography (GC) and liquid chromatography (LC) [II]. These techniques have both strengths and weaknesses as shown in Table I.

Table 1. Nuclear magnetic resonance (NMR) and mass spectroscopy (MS) advantages and disadvantages.

\begin{tabular}{lll}
\hline Technique & Advantages & Disadvantages \\
\hline NMR & Non-destructive & Low sensitivity \\
& $\begin{array}{l}\text { Minimal sample preparation } \\
\text { (chromatography/analyte ionization is not needed) }\end{array}$ \\
& Quantitative (a large number of metabolites) & \\
\hline MS & High sensitivity & Poor analytical productivity \\
& Dynamic rage & Destructive (metabolite identification is based on \\
& & fragmentation patterns) \\
& & Isotopic standards required for absolute quantification \\
\hline
\end{tabular}

\section{Nuclear Magnetic Resonance Spectroscopy (NMR)}

NMR is an appealing technique used in conducting metabolomics research because it offers a rapid, non-destructive, and quantitative analysis. NMR is a technique able to identify the structure and to quantify metabolites using the magnetic characteristics of a nucleus. The nucleus placed into a strong magnetic field, at a specific frequency, can absorb electromagnetic radiations. The nature of surrounding atoms characteristically influences the signal of NMR. Subsequently, it produces chemical shifts able to assign a local molecular structure. The advantage of this technique arises from the absence of chromatographic separation and ionization of samples and its ability to offer significant information about the metabolic pathways. Subsequently, NMR technique is non-destructive and requires a low time for sample preparation. The main disadvantage of using NMR for metabolomic analysis is the requirement for manual spectral profiling and the limitation of metabolites abundance, typically less than roo analytes coming from human samples [I2].

\section{Mass Spectrometry (MS)}

MS is a high analytical technique used to measure small molecules and proteins. The first step in a metabolite profiling study is to separate analytes by using techniques like gas chromatography/MS (GC/MS), liquid chromatography/MS (LC/MS), or capillary electrophoresis/MS (CE/MS). In contrast with LC, which is used to separate non-volatile metabolites in a solution, GC is used to evaluate volatile (non-polar) metabolites. Performing efficient desorption and ionization of metabolites is necessary for an accurate MS-based approach. Consequently, gas-phase ions are divided by mass analyses like quadrupole time-of-flight MS (QTOF-MS) and ion-trap MS [13].

By using microchannel plates and a photomultiplier tube the ions can be detected. The ion is recognized by comparing the exact mass, the fragmented information obtained, and the retention time with spectral data and original standards. As the name implies, MS identifies metabolites depending on the mass-to-charge ratio $(\mathrm{m} / \mathrm{z})$ of their respective ions $[\mathrm{I} 4]$. 
TOF-MS accelerates ions by applying a fixed electronic field, and then measures the time the ion needs to reach the detector. The first ion that will arrive at the detector is the one with the lowest mass. The biggest advantage of TOF-MS includes a wide mass-to-charge range and excellent mass accuracy [ $\left.\mathrm{r}_{3}\right]$.

Quadruple-mass spectrometry serves as a mass-selective filter that contains four parallel rods equidistantly arranged from a central axis. In this technique, the ion with a specific $\mathrm{m} / \mathrm{z}$ value will get to the detector because of its stable trajectory. The quadrupole-MS has a better linear dynamic range in the relative quantification as compared to TOF-MS. However, TOF-MS is superior to quadrupole-MS instruments like mass accuracy, also to $\mathrm{m} / \mathrm{z}$ range. Nevertheless, triple-quadruple instruments can perform in tandem with MS spectrometry, improving the analytical specificity and sensitivity for designated metabolites [I4].

Ion trap-MS forms a trap for a specific metabolite by applying various configurations to an electrical field, where a selected mass-to-charge ratio ion can be held and can accumulate. The metabolite trapped can be ejected from the ion trap or can be fragmented inside it by means of MS analysis in tandem with ion trap-MS. This method can magnify the analytical sensitivity providing a less robust quantification in a more dynamic and small range [15].

\section{Chronic Kidney Disease (CKD)}

The kidney has an essential role in maintaining physiological homeostasis being an essential organ in the excretion of toxic solutes, hormone production, and blood pressure regulation. It also maintains ionic, osmotic, and $\mathrm{pH}$ balance. The incidence of kidney diseases has been in a continuous increase, affecting nowadays a significant proportion of the global population. The effects that kidney diseases have on the social system and economics are enormous because the treatment is often invasive and expensive. Therefore, modern medicine has as primary purpose an effective diagnosis and treatment [16].

CKD is becoming rapidly a significant health problem globally. The overall prevalence of CKD (stage I-5) is estimated worldwide at $13.4 \%$ and it is constantly increasing [17]. The underlying mechanisms of renal impairment progression are hemodynamic factors, proteinuria, dyslipidemia, podocyte loss, growth factors, cytokines, and the renin-angiotensin-aldosterone system [18].

Metabolomics research in nephrology has started from uremia analyses and has advanced to longitudinal and cross-sectional studies. The primer focus is on discovering new biomarkers in the early stages of kidney disease. Thus, it is important to find new biomarkers able to prevent the early decrease in kidney function within the general population.

\section{Biomarkers}

The National Institute of Health Biomarkers Definition Working Group defines a biomarker as "a characteristic that is objectively measured and evaluated as an indicator of normal biologic processes, pathogenic processes, and pharmacologic responses to therapeutic intervention" [ $\left.{ }_{17}\right]$. Every type of cell and living organisms possesses a genetically predefined collection of metabolites under particular environmental conditions. These metabolites oscillate under physiologic and pathologic conditions. Recent metabolomics studies have identified metabolic biomarkers, providing data about the underlying mechanisms in various diseases (obesity, cardiovascular diseases, CKD and diabetes mellitus) [19]. The metabolites studied in CKD are presented in Table 2. 
Table 2. Summary of studies in the field of metabolomics and chronic kidney disease (CKD).

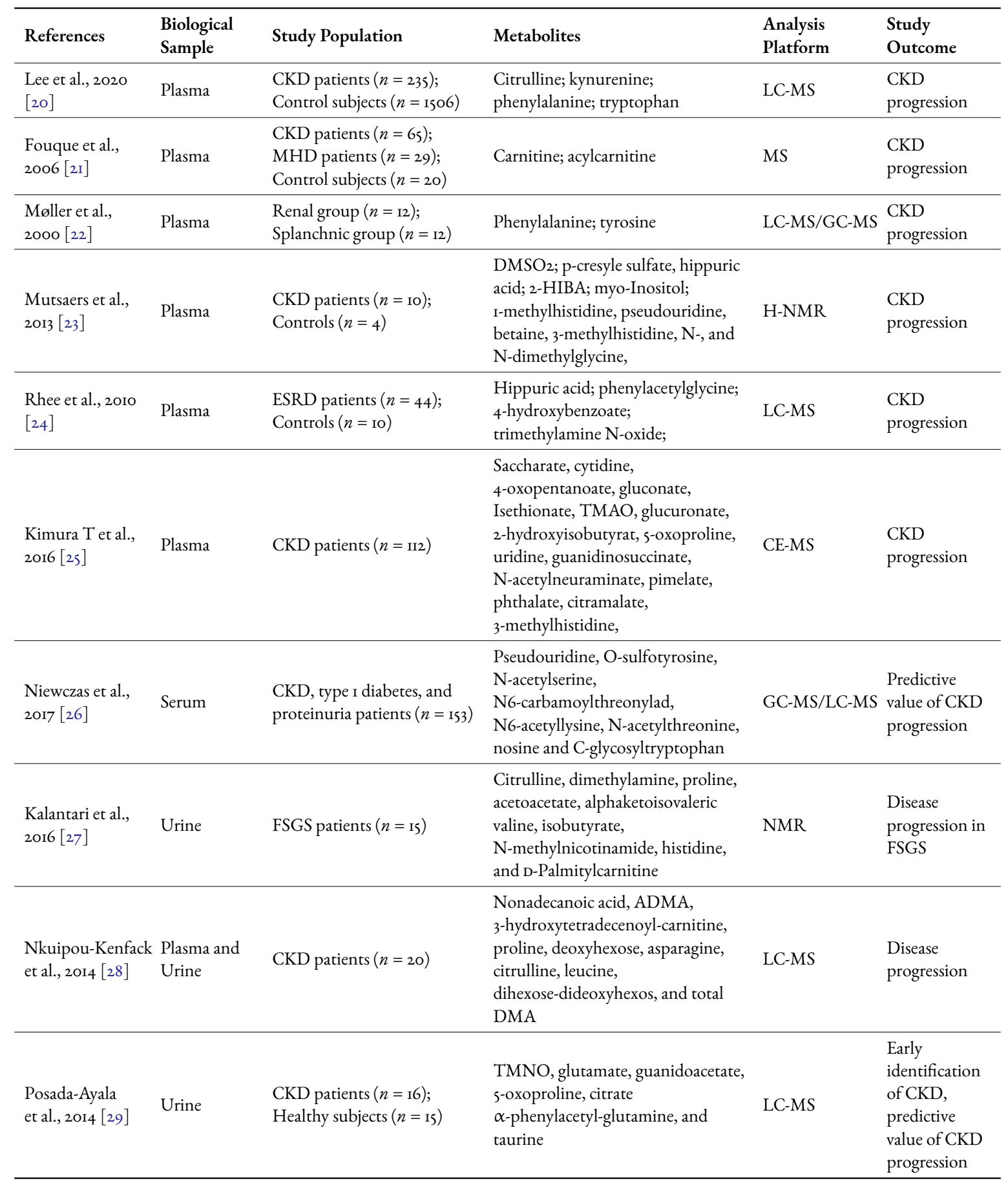

CKD: chronic kidney disease; LC-MS: liquid chromatography-mass spectrometry; GC-MS: gas chromatography-mass spectrometry; NMR: nuclear magnetic resonance spectroscopy; CE-MS: capillary electrophoresis-mass spectrometry; ESRD: end-stage renal disease; $\mathrm{DMSO}_{2}$ : dimethyl sulfone; 2-HIBA: 2-hydroxyisobutyric acid; ADMA: Asymmetric dimethylarginine; total DMA: total dimethylarginine; TMNO: trimethylamine N-oxide.

\section{Experimental Studies}

In this section, we will briefly review a series of plasmatic and urinary metabolites. 
Lee et al. identified 28 new metabolites in a follow-up cohort study performed on the Korean population. Interestingly, this study established a correlation between estimated glomerular filtration rate (eGFR) and the clinical outcomes of metabolites in CKD, as compared with previous studies [20].

The most significant metabolites which correlate with CKD development were citrulline, kynurenine, and the kynurenine: tryptophan ratio [30].

Concomitantly, the KORA study showed a relation between acylcarnitine levels and eGFR decrease. The increase in the plasmatic level of acylcarnitine is associated with a reduced excretion in patients with altered renal function [31]. Most likely, the amino acids have an essential role in the pathogenesis of kidney disease. The mechanisms implicated are inhibition of endothelial nitric oxide synthesis, oxidative stress, or enzymatic activity [32].

Amino acids are essential for metabolic processes. By generating oxidative stress (methionine sulfoxide/methionine) and nitric oxide production (citrulline/arginine) these have a role in kidney function decline [32].

The arginine homeostasis is sustained by a series of metabolic interactions between the bowel, liver, and kidney. The main site of citrulline synthesis is the intestine, where amino acids enter the circulation to be taken up by the kidney. Next, the kidney, in the convoluted tubule, converts the citrulline to arginine. The main enzymes involved in this process are cytosolic enzymes arginine succinate synthetase and arginine succinate lyase [33]. Tizianello et al. studied the arteriovenous renal gradient of amino acid in healthy subjects and patients suffering from CKD. Their results showed that the citrulline uptake was equal with the arginine output, the last one being with $40 \%$ less in patients with CKD as compared with healthy subjects [34].

Multiple studies have shown that citrulline levels correlate with CKD prevalence and incidence in patients with $\mathrm{CKD}_{\text {, stage }} \mathrm{G}_{3}-\mathrm{G}_{5}$ by KDIGO (the Kidney Disease Improving Global Outcomes) classification. The citrulline is generated in the arginine-succinate pathway from the arginine and urea cycle from carbamoyl phosphate and ornithine. This metabolite seems to correlate with both prevalence and incidents in CKD patients [33].

Another metabolite, like kynurenine, is associated with the progression and prediction of CKD. L-tryptophane, present in the blood, is metabolized by the liver to L-Kynurenine along the kynurenine pathways by hepatic enzyme L-tryptophane 2,3-dioxygenase (TDO) and extrahepatic enzyme indoleamine 2,3-deoxygenase (IDO), the final products eliminated by the kidney. The levels of serum L-tryptophane in patients with CKD are significantly increased and seem to be parallel with the severity of the disease. Increased serum L-kynurenine correlates with high levels of TDO and reduced kynurenines activity in the liver [35].

Increased plasmatic levels of kynurenine are associated with a decline in kidney function. The kynurenine: tryptophan ratio seems to be a predictor of CKD. It is well known that the kynurenine: tryptophan ratio reflects the IDO activity. Recent studies have shown a correlation between the early stage of CKD and IDO activity. Particularly, high levels were correlated with the disease severity and inflammatory markers like hsCRP (high-sensitivity C-reactive protein) and sTNFR-I (soluble tumor necrosis factor receptor-I). The main reason for high IDO levels is chronic inflammation, a characteristic feature for CKD [36].

Interestingly, tryptophan (TRP) and its metabolites play an essential role in the regulation of adaptive immunity. Additionally, this can lead to atherosclerosis, a chronic complication of CKD. 
The TRP metabolites are kynurenic acid (KYHA), 3-hydroxykynurenine, and quinolinic acid (QA). Patients suffering from CKD seem to be at a high risk of KYNA-associated diseases. It has been hypothesized that TRP activates oxidative stress and the migration of leukocytes into vessel cells, such as vascular smooth muscle cells and endothelial cells. Proinflammatory cytokines are the principal source of IDO. In chronic inflammation there is observed an increased production of IFN-y (interferon-gamma) and an increased tryptophan metabolism [37]. Therefore, the accumulation of these metabolites in the plasma and tissues contributes to the decrease in GFR. In CKD patients are observed normal or decreased levels of metabolites for the kynurenine pathway, kynurenines, kynurenine aminotransferase, ACMSD (human- $\alpha$-amino- $\beta$-carboxymuconate- $\varepsilon$-semialdehyde decarboxylase), and 3 -hydroxyanthranilate-3,4-dioxygenase. At variance, IDO, TDO, and kynurenine 3-monooxygenase have increased activity in CKD patients [38].

Phenylalanine is a vital amino acid that in humans is degraded to tyrosine and synthesized by an enzyme called phenylalanine 4-hydroxylase. Tyrosine is a semi-essential amino acid. The deficiency of tyrosine affects the remodeling of body proteins. The kidney and the splanchnic bed (presumably the liver) are the sites of phenylalanine-tyrosine conversion. However, the principal source of circulating tyrosine is the kidney. The levels of phenylalanine in patients with CKD are normal or slightly increased. In contrast, it is observed a decrease in plasmatic or skeletal muscle concentration of tyrosine levels and an increase in phenylalanine: tyrosine ratio [22].

The accumulation of uremic toxins is a hallmark for CKD. Mutsaers et al. reported for the first-time high plasma levels of $\mathrm{DMSO}_{2}$ (dimethyl sulfone) and 2-HIBA (2-hydroxyisobutyric acid) in patients with CKD. Moreover, this study showed elevated levels of p-cresyle sulfate, hippuric acid, myo-Inositol, I-methylhistidine, 3-methylhistidne, $N, N$-dimethylglycine, pseudouridine, and betaine in stage $\mathrm{G}_{3}-\mathrm{G}_{4}$ of CKD patients [23].

$\mathrm{DMSO}_{2}$, a uremic metabolite, was firstly described in 1966 by William et al. in human urine. It seems to originate from dietary sources (milk and port wine). Recent studies have shown that $\mathrm{DMSO}_{2}$ can reduce the production of Interleukin-6 and Interleukin-8 in the human chondrocyte cell line ( $\left.\mathrm{C}_{2} \mathrm{OA} 4\right)$, probably by inhibiting the ERKI/2 signaling pathway (extracellular signal-regulated protein kinases I and 2) [23]. In a study performed on nephrectomy rats with CKD, insignificant differences in $\mathrm{DMSO}_{2}$ levels were observed, but the plasmatic and urinary levels seemed to defer in an advanced CKD stage [39]. Mika et al. founded elevated levels of DMSOS in stage $\mathrm{G}_{3 \mathrm{a}}$ and $\mathrm{G}_{3 \mathrm{~b}}$ CKD patients. The increase in plasmatic levels was linked with a reduction in $\mathrm{DMSO}_{2}$ excretion [40].

2-HIBA is an essential metabolite in both urine and serum. Interestingly, 2-HIBA plays a role in type-2 diabetes mellitus mechanism by alteration in fatty acid metabolism [23].

In addition, Rhee et al. performed a study on 44 patients undergoing hemodialysis by using LC-MS-based metabolite profiling. They demonstrated the retention of other metabolites such as hippuric acid, phenylacetylglycine, 4-hydroxybenzoate, and trimethylamine $\mathrm{N}$-oxide. The production of the metabolites was a result of bacterial metabolism in gastrointestinal tract [24].

Several studies have reported a series of new urinary metabolites in CKD patients. McMahon et al. evaluated the correlation between new urinary metabolites (glycine and histidine) and CKD. Additionally, by genomic technologies, they showed an association between $\mathrm{SLC}_{7} \mathrm{~A}_{9}$ and kidney disease. Nevertheless, 
these studies identified a connection between urinary lysine and NMMA (monomethylated derivative), and CKD patients [4I]. These results were confirmed in the ARIC cohort [42].

Glycine, a semi-essential amino acid, has a key role in glyoxylate metabolism and the alanine-glyoxylate defect. The effects exerted by glycine could be the development of type I hyperoxaluria, kidney stones, and CKD. At the same time, glycine can reduce $\mathrm{T}$-cell proliferation and proinflammatory cytokines production in vitro. Glycine stimulates receptors called N-methyl-D-aspartate, located in the proximal tubule. Consequently, glycine produces oxide-dependent vasodilatation and increases single-nephron GFR [4I].

Histidine, an essential amino acid, has a protective mechanism against oxidative stress. A new study performed on patients with CKD correlated the increase in mortality and oxidative stress with decreased plasma levels in histidine [4I].

Glutamate, "a non-essential amino acid”, is a precursor of glutamine, resulting in ammonia production by 5 -oxiproline. It has been demonstrated that in patients with CKD and metabolic acidosis glutamate acts as an inhibitor of glutamine deamidating enzyme and increases ammonia production. In conclusion, in CKD patients it can be observed a low urinary level of 5-oxiproline and an increase in glutamate levels [25].

ADMA (asymmetric dimethylarginine) is produced from intracellular proteins by regular proteolysis. While a small amount of ADMA is excreted in the urine, the majority of ADMA, approximately 90\%, is hydrolyzed to DMA (dimethylamine) and L-citrulline. DMA is frequently found in the urine. A decrease in GFR has been correlated with a low urinary ADMA [28].

Trimethylamine-N-oxide (TMNO) is a plasmatic amine, produced by the gut flora from different foods or dietary phosphatidylcholine as TMA (trimethylamine) and finely excreted in urine as TMNO. One study showed increased urinary levels in patients with CKD and increased plasmatic levels in patients under dialysis [29].

\section{Conclusions}

Metabolomics study in CKD patients is a promising field that may provide an innovative approach to an early diagnosis, disease progression, and treatment of renal diseases. Moreover, it offers a better comprehension of pathogenesis, renal metabolite handling, and cellular metabolism within the kidney.

Personalized medicine is a novel approach to traditional medicine, focused on specific characteristics of a patient. The metabolomics techniques integrated with modern multivariate data analysis series have led to new prognostic and diagnostic biomarkers. The integration of these biomarkers into clinical research and practice will identify even minor differences in pathological conditions.

Nephrology is a vast domain with an increased interest in metabolomics profiles. Establishing and comparing metabolomics fingerprints patterns of a specific metabolite is the next step concomitantly with the observation of changes which occur as a response to drug exposure or diseases. Validation of metabolomic biomarkers in CKD patients requires the conduction of epidemiological cohort studies.

Author Contributions: Conceptualization, M.-R.P., L.P., D.M.M. and C.S.; Validation, L.P., D.M.M. and C.S.; Formal Analysis, A.V.; Investigation, A.S.; Resources, L.B.; Data Curation, S.I.; Writing-Original Draft Preparation, M.-R.P.; Writing-Review and Editing, L.P., D.M.M. and C.S.; Supervision, F.G.; Project Administration, D.C.J.; Funding Acquisition, M.-R.P. 
Funding: This work has been supported by the Victor Babeș University of Medicine and Pharmacy Timișoara, Internal Grant for Ph.D. Students, code 6DOC/1865/12.02.2020 (M.R.P).

Conflicts of Interest: The authors declare no conflict of interest.

\section{References}

I. Micheel, C.M.; Nass, S.J.; Omenn, G.S. (Eds.) Omics-Based Clinical Discovery: Science, Technology, and Applications; National Academies Press: Washington, DC, USA, 2012.

2. Lopez, A.; Murray, C. The global burden of disease, 1990-2020. Nat. Med. 1998, 4, I24I-I243. [CrossRef] [PubMed]

3. Patti, G.J.; Yanes, O.; Siuzdak, G. Metabolomics: Beyond biomarkers and towards mechanisms. Nat. Rev. Mol. Cell Biol. 2016, 17, 45I-459.

4. Collins, F.S.; McKusick, V.A. Implications of the Human Genome Project for Medical Science. JAMA 2001, 285, 540-544. [CrossRef] [PubMed]

5. Wishart, D.S.; Knox, C.; Guo, A.C.; Eisner, R.; Young, N.; Gautam, B.; Hau, D.D.; Psychogios, N.; Dong, E.; Bouatra, S.; et al. HMDB: A knowledgebase for the human metabolome. Nucleic Acids Res. 2009, 37, D603-D6io. [CrossRef] [PubMed]

6. Sun, J.; Beger, R.D.; Schnackenberg, K.L. Metabolomics as a tool for personalizing medicine: 2012 update. Pers. Med. 2013, 10, I49-16I. [CrossRef] [PubMed]

7. Patti, G.; Yanes, O.; Siuzdak, G. Metabolomics: The apogee of the omics trilogy. Nat. Rev. Mol. Cell Biol. 2012, 13, 263-269. [CrossRef] [PubMed]

8. Orešič, M. Metabolomics, a novel tool for studies of nutrition, metabolism and lipid dysfunction. Nutr. Metabol. Cardiovasc. Dis. 2009, 19, 816-824. [CrossRef] [PubMed]

9. Wishart, D.S.; Feunang, Y.D.; Marcu, A.; Guo, A.C.; Liang, K.; Vázquez-Fresno, R.; Sajed, T.; Johnson, D.; Li, C.; Karu, N.; et al. HMDB 4.0: The human metabolome database for 2018. Nucleic Acids Res. 2018, 46, D6o8-D6r7. [CrossRef] [PubMed]

Io. Ribbenstedt, A.; Ziarrusta, H.; Benskin, J.P. Development, characterization and comparisons of targeted and non-targeted metabolomics methods. PLOS ONE 2018, 13, e0207082. [CrossRef] [PubMed]

II. Zhang, X.; Zhu, X.; Wang, C.; Zhang, H.; Cai, Z. Non-targeted and targeted metabolomics approaches to diagnosing lung cancer and predicting patient prognosis. Oncotarget 2016, 7, 63437-63448. [CrossRef]

I2. Emwas, A.H.; Roy, R.; McKay, R.T.; Tenori, L.; Saccenti, E.; Gowda, G.A.; Raftery, D.; Alahmari, F.; Jaremko, L.; Jaremko, M.; et al. NMR Spectroscopy for Metabolomics Research. Metabolites. 2019, 9, 123. [CrossRef]

I3. Plumb, R.S.; Stumpf, C.L.; Gorenstein, M.V.; Castro-Perez, J.M.; Dear, G.J.; Anthony, M.; Sweatman, B.C.; Connor, S.C.; Haselden, J.N. Metabonomics: The use of electrospray mass spectrometry coupled to reversed-phase liquid chromatography shows potential for the screening of rat urine in drug development. Rapid Commun. Mass Spectrom. 2002, 16, 1991-1996. [CrossRef]

I4. Hirayama, A.; Wakayama, M.; Soga, T. Metabolome analysis based on capillary electrophoresis-mass spectrometry. Trends Anal. Chem. 2014, 61, 215-222. [CrossRef]

15. Rhee, E.P.; Gerszten, R.E. Metabolomics and Cardiovascular Biomarker Discovery. Clin. Chem. 2012, 58, I39-I47. [CrossRef]

16. Clark, A.; Jit, M.; Warren-Gash, C.; Guthrie, B.; Wang, H.H.; Mercer, S.W.; Sanderson, C.; McKee, M.; Troeger, C.; Ong, K.L.; et al. Global, regional, and national estimates of the population at increased risk of severe COVID-I9 due to underlying health conditions in 2020: A modelling study. Lancet Glob. Health 2020, 8, егоo3-еiоr7. [CrossRef]

17. Hill, N.R.; Fatoba, S.T.; Oke, J.L.; Hirst, J.A.; O’Callaghan, C.A.; Lasserson, D.S.; Hobbs, F.R. Global Prevalence of Chronic Kidney Disease-A Systematic Review and Meta-Analysis. PLoS ONE 2016, 11, eois8765. [CrossRef]

18. Fogo, A.B. Mechanisms of progression of chronic kidney disease. Pediatr. Nephrol. 2007, 22, $201 \mathrm{I}-2022$. [CrossRef]

19. Strimbu, K.; Tavel, J.A. What are biomarkers? Curr. Opin. HIV AIDS 2010, 5, 463-466. [CrossRef]

20. Lee, H.; Jang, H.B.; Yoo, M.G.; Park, S.I.; Lee, H.J. Amino Acid Metabolites Associated with Chronic Kidney Disease: An Eight-Year Follow-Up Korean Epidemiology Study. Biomedicines 2020, 8, 222. [CrossRef]

2I. Fouque, D.; Holt, S.; Guebre-Egziabher, F.; Nakamura, K.; Vianey-Saban, C.; Hadj-Aïssa, A.; Hoppel, C.L.; Kopple, J.D. Relationship Between Serum Carnitine, Acylcarnitines, and Renal Function in Patients with Chronic Renal Disease. J. Ren. Nutr. 2016, 16, I25-131. [CrossRef]

22. Møller, N.; Meek, S.; Bigelow, M.; Andrews, J.; Nair, K.S. The kidney is an important site for in vivo phenylalanine-to-tyrosine conversion in adult humans: A metabolic role of the kidney. Proc. Natl. Acad. Sci. USA 2000, 97, 1242-1246. [CrossRef] [PubMed] 
23. Mutsaers, H.A.; Engelke, U.F.; Wilmer, M.J.; Wetzels, J.F.; Wevers, R.A.; van den Heuvel, L.P.; Hoenderop, J.G.; Masereeuw, R. Optimized Metabolomic Approach to Identify Uremic Solutes in Plasma of Stage 3-4 Chronic Kidney Disease Patients. PLoS ONE 2013, 8, e7I199. [CrossRef] [PubMed]

24. Rhee, E.P.; Souza, A.; Farrell, L.; Pollak, M.R.; Lewis, G.D.; Steele, D.J.; Thadhani, R.; Clish, C.B.; Greka, A.; Gerszten, R.E. Metabolite profiling identifies markers of uremia. J. Am. Soc. Nephrol. 2010, 21, I04I-IO5I. [CrossRef] [PubMed]

25. Kimura, T.; Yasuda, K.; Yamamoto, R.; Soga, T.; Rakugi, H.; Hayashi, T.; Isaka, Y. Identification of biomarkers for development of end-stage kidney disease in chronic kidney disease by metabolomic profiling. Sci. Rep. 2016, 6, 26138. [CrossRef]

26. Niewczas, M.A.; Mathew, A.V.; Croall, S.; Byun, J.; Major, M.; Sabisetti, V.S.; Smiles, A.; Bonventre, J.V.; Pennathur, S.; Krolewski, A.S. Circulating modified metabolites and a risk of esrd in patients with type i diabetes and chronic kidney disease. Diabetes Care 2017, 40, 383-390. [CrossRef]

27. Kalantari, S.; Nafar, M.; Samavat, S.; Parvin, M.; Nobakht, M.G.H.B.F.; Barzi, F. NMR-based metabolomics exploring urinary biomarkers correlated with proteinuria in focal segmental glomerulosclerosis: A pilot study: Urine metabolomics based on NMR. Magn. Reson. Chem. 2016, 54, 821-826. [CrossRef]

28. Nkuipou-Kenfack, E.; Duranton, F.; Gayrard, N.; Argilés, À.; Lundin, U.; Weinberger, K.M.; Dakna, M.; Delles, C.; Mullen, W.; Husi, H.; et al. Assessment of Metabolomic and Proteomic Biomarkers in Detection and Prognosis of Progression of Renal Function in Chronic Kidney Disease. PLoS ONE 2014, 9, e96955. [CrossRef]

29. Posada-Ayala, M.; Zubiri, I.; Martin-Lorenzo, M.; Sanz-Maroto, A.; Molero, D.; Gonzalez-Calero, L.; Fernandez-Fernandez, B.; De La Cuesta, F.; Laborde, C.M.; Barderas, M.G.; et al. Identification of a urine metabolomic signature in patients with advanced-stage chronic kidney disease. Kidney Int. 2014, 85, I03-III.

30. Hanna, M.H.; Brophy, P.D. Metabolomics in pediatric nephrology: Emerging concepts. Pediatr. Nephrol. 2015, 30, 88I-887. [CrossRef]

3I. Holle, R.; Happich, M.; Löwel, H.; Wichmann, H.E.; MONICA/KORA Study Group. KORA-A research platform for population based health research. Gesundheitswesen 2005, 67 (Suppl. I), Si9-S25. [CrossRef]

32. Abbiss, H.; Maker, G.L.; Trengove, R.D. Metabolomics Approaches for the Diagnosis and Understanding of Kidney Diseases. Metabolites 2019, 9, 34. [CrossRef]

33. Lau, T.; Owen, W.; Yu, Y.M.; Noviski, N.; Lyons, J.; Zurakowski, D.; Tsay, R.; Ajami, A.; Young, V.R.; Castillo, L. Arginine, citrulline, and nitric oxide metabolism in end-stage renal disease patients. J. Clin. Investig. 2000, 105, I217-I225. [CrossRef]

34. Tizianello, A.; De Ferrari, G.; Garibotto, G.; Gurrei, G.; Robaudo, C. Renal metabolism of amino acids and ammonia in subjects with normal renal function and in patients with chronic renal insufficiency. J. Clin. Investig. 1980, 65, II62-II73.

35. Saito, K.; Fujigaki, S.; Heyes, M.P.; Shibata, K.; Takemura, M.; Fujii, H.; Wada, H.; Noma, A.; Seishima, M. Mechanism of increases in L-kynurenine and quinolinic acid in renal insufficiency. Am. J. Physiol. Ren. Physiol. 2000, 279, F565-F572. [CrossRef]

36. Schefold, J.C.; Zeden, J.P.; Fotopoulou, C.; von Haehling, S.; Pschowski, R.; Hasper, D.; Volk, H.D.; Schuett, C.; Reinke, P. Increased indoleamine 2,3-dioxygenase (IDO) activity and elevated serum levels of tryptophan catabolites in patients with chronic kidney disease: A possible link between chronic inflammation and uraemic symptoms. Nephrol. Dial. Transplant. 2009, 24, 190I-1908. [CrossRef]

37. Debnath, S.; Velagapudi, C.; Redus, L.; Thameem, F.; Kasinath, B.; Hura, C.E.; Lorenzo, C.; Abboud, H.E.; O'Connor, J.C. Tryptophan Metabolism in Patients with Chronic Kidney Disease Secondary to Type 2 Diabetes: Relationship to Inflammatory Markers. Int. J. Tryptophan Res. 2017, 10. [CrossRef]

38. Mor, A.; Kalaska, B.; Pawlak, D. Kynurenine Pathway in Chronic Kidney Disease: What's Old, What's New, and What's Next? Int. J. Tryptophan Res. 2020, 13, I-I8. [CrossRef]

39. Hanifa, M.A.; Skott, M.; Maltesen, R.G.; Rasmussen, B.S.; Nielsen, S.; Frøkiær, J.; Ring, T.; Wimmer, R. Tissue, urine and blood metabolite signatures of chronic kidney disease in the $5 / 6$ nephrectomy rat model. Metabolomics 2019, 15, in2. [CrossRef]

40. Mika, A.; Wojtowicz, W.; Ząbek, A.; Młynarz, P.; Chmielewski, M.; Sledzinski, T.; Stepnowski, P. Application of nuclear magnetic resonance spectroscopy for the detection of metabolic disorders in patients with moderate kidney insufficiency. J. Pharm. Biomed. Anal. 2018, 149, I-8. [CrossRef]

4I. McMahon, G.M.; Hwang, S.J.; Clish, C.B.; Tin, A.; Yang, Q.; Larson, M.G.; Rhee, E.P.; Li, M.; Levy, D.; O'Donnell, C.J.; et al. Urinary metabolites along with common and rare genetic variations are associated with incident chronic kidney disease. Kidney Int. 2017, 91, I426-I435. [CrossRef]

42. Yu, B.; Zheng, Y.; Nettleton, J.A.; Alexander, D.; Coresh, J.; Boerwinkle, E. Serum metabolomic profiling and incident CKD among African Americans. Clin. J. Am. Soc. Nephrol. 2014, 9, I4IO-I4I7. [CrossRef] [PubMed]

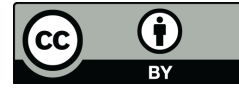

